Jean-François Dortier, Les sciences humaines. Panorama des connaissances

Éditions Sciences Humaines/Paris, 2001, 487 p.

\title{
Alain Kimmel
}

\section{OpenEdition}

\section{Journals}

Édition électronique

URL : https://journals.openedition.org/ries/1795

DOI : 10.4000/ries. 1795

ISSN : 2261-4265

Éditeur

France Education international

Édition imprimée

Date de publication : 1 avril 2002

Pagination : 22-24

ISBN : 2-84520-553-8

ISSN : 1254-4590

\section{Référence électronique}

Alain Kimmel, « Jean-François Dortier, Les sciences humaines. Panorama des connaissances », Revue internationale d'éducation de Sèvres [En ligne], 29 | avril 2002, mis en ligne le 01 avril 2005, consulté le 05 juillet 2021. URL : http://journals.openedition.org/ries/1795 ; DOI : https://doi.org/10.4000/ries. 1795

Ce document a été généré automatiquement le 5 juillet 2021

(c) Tous droits réservés 


\section{Jean-François Dortier, Les sciences humaines. Panorama des connaissances}

Éditions Sciences Humaines/Paris, 2001, 487 p. Alain Kimmel

\section{RÉFÉRENCE}

Les sciences humaines. Panorama des connaissances. Jean-François Dortier. Éditions Sciences Humaines/Paris, 2001, 487 p.

1 Le domaine des sciences de l'homme est notamment exploré depuis une dizaine d'années par un magazine qui a pris pour titre Sciences humaines. Né en novembre 1990, ce magazine a publié à ce jour 122 numéros mensuels et 34 numéros hors série. Dans chacune de ces publications, on peut trouver des synthèses sur les acquis et les débats en sciences humaines et sociales à travers des dossiers, des articles, des échos des recherches et des recensions d'ouvrages.

Devant le succès rencontré par la revue, ses animateurs ont créé une collection d'ouvrages de synthèse qui présentent l'état des connaissances dans les principales disciplines des sciences de l'homme et de la société. Ces livres collectifs comportent des articles publiés dans le magazine ou dans les hors séries, mais actualisés et complétés par de nouvelles contributions. Dix titres ont été publiés, dont celui, entièrement inédit, intitulé Les sciences humaines.

Douze disciplines sont abordées dans ce livre de près de 500 pages: anthropologie, linguistique, psychologie, psychopathologie, sciences cognitives, psychologie sociale, sociologie, économie, préhistoire, histoire, géographie, philosophie. Son objectif est d'offrir un panorama des principales théories et hypothèses dans chacun de ces domaines.

4 «Il n'y a pas de question importante dont la solution ne soit comprise dans la science de l'homme» écrivait David Hume en 1793, tandis que d'Alembert proposait un «programme pour la science de l'homme». Ce n'est cependant qu'au début du $\mathrm{XX}^{\mathrm{e}}$ 
siècle que seront tracées les grandes frontières disciplinaires, avec leurs chaires universitaires, leurs sociétés savantes et leurs revues. Tout au long du siècle, les sciences humaines vont se développer de manière exponentielle. En France actuellement, on compte quelque 20000 chercheurs (dont 2000 au CNRS) et enseignants-universitaires, il paraît chaque année environ 4000 livres, sans compter la « littérature grise ».

Comme le souligne J.-F. Dortier, dans son introduction, « les sciences humaines forment un vaste univers de connaissances à la fois riche, varié et ... désordonné ». Il ne peut être bien sûr question, dans ce cadre, de pénétrer en profondeur dans cet univers. Tentons quand même quelques brèves incursions dans certains des domaines abordés, en sachant que chaque discipline est globalement traitée selon le même schéma : une problématique, des définitions, un historique, quelques grands thèmes ou disciplines annexes.

6 Ainsi pour l'anthropologie, chapitre sous-titré «Au cœur des sociétés », sont présentés ce qui la différencie de l'ethnologie, les notions d'anthropologie culturelle et sociale, les différents courants, les notions de parenté, pouvoir, religion...

7 Le chapitre sur la linguistique («Les secrets du langage») traite de la nature, de l'origine et de l'histoire des langues, du langage et de son fonctionnement, de la parole et de ses usages, de la communication et de ses mystères. Comme dans tous les chapitres, on trouve des encadrés fort utiles qui rappellent les données de base de la discipline, ici les différents domaines de la linguistique, les parentés et influences entre les langues, la sémiologie, etc.

8 La psychologie ou « Les mystères du psychisme » est un des chapitres les plus riches. Il s'efforce de nous introduire dans "Les arcanes de la pensée ", avec en particulier un encadré très dense sur la question sans cesse reformulée de l'héritabilité de l'intelligence. Il aborde également l'immense domaine de la vie affective et celui des diverses approches en psychologie.

9 Le chapitre sur les sciences cognitives («Du cerveau à l'esprit ») est certainement très attendu par les lecteurs, compte tenu de la place de plus en plus importante qu'occupent ces sciences depuis quelques années. Il s'ouvre sur la notion capitale de " révolution cognitive ", avec ses cinq pôles disciplinaires (psychologie, neurosciences, linguistique, philosophie de l'esprit et intelligence artificielle). Il présente les modèles de la pensée, examine le trajet du cerveau à l'ordinateur, avec des encadrés passionnants sur l'univers des neurosciences et sur les réseaux sémantiques.

10 La sociologie («La construction des sociétés ») est probablement un des chapitres qui intéressera le plus le lecteur non spécialiste, car cette discipline est aussi riche que diverse par la multiplicité de ses théories, de ses modèles, de ses méthodes ou démarches, de ses applications (sociologie de l'éducation, de la famille, du travail, des religions, des loisirs...), de ses écoles et bien sûr de ses grands auteurs qui furent souvent plus que de "simples» sociologues (Comte, Marx, Tocqueville, Durkheim, Mauss, Weber, Simmel, etc.).

11 L'histoire ("À la recherche du passé ») est également un chapitre qui nous amène à la rencontre d'auteurs qui, par l'ampleur et le rayonnement de leur pensée, dépassent le cadre strict de cette discipline et atteignent au niveau le plus élevé du statut de " grand intellectuel » (Arnold Toynbee, Fernand Braudel, Michel Foucault, par exemple). La question «À quoi sert l'histoire? » est posée, de même que celles de ses rapports avec 
la mémoire nationale, de la notion d'histoire-récit, du métier d'historien, des grands chantiers de l'histoire et des différentes manières de « faire de l'histoire ».

12 Le dernier chapitre du livre est consacré à la philosophie ("À la recherche du sens »). Il présente «trois visages» de cette discipline sous forme d'une triple interrogation: «Un questionnement critique?», «Une quête de vérité ? ", «Un art de vivre? ». Sous la rubrique "Philosophie de la connaissance» sont présentées les théories de l'empirisme, du subjectivisme et de la phénoménologie. Les autres rubriques abordées sont celles de la «Philosophie morale » et de la «Philosophie politique». La question classique « Qu'est-ce que l'homme ? » est posée en préalable à l'examen des différentes conceptions de la nature humaine (rationaliste, naturaliste, utilitariste, prométhéenne, vitaliste, existentialiste, etc.). Après un rappel des principaux courants philosophiques $\mathrm{du} \mathrm{XX}^{\mathrm{e}}$ siècle, le chapitre se clôt sur les «interrogations contemporaines" de la philosophie d'aujourd'hui.

13 À la fois guide de lecture, encyclopédie et ouvrage de référence, ce livre est tout à fait fidèle à l'esprit de la revue Sciences humaines, celui d'une vulgarisation sérieuse, intelligente et de grande qualité.

\section{AUTEURS}

\section{ALAIN KIMMEL}

Chargé de programmes, CIEP 